\title{
Alleviation of atopic dermatitis-related symptoms by Perilla frutescens Britton
}

\author{
JIN-CHUL HEO ${ }^{1,4^{*}}$, DONG-YOON NAM ${ }^{2 *}$, MYUNG SUN SEO ${ }^{3}$ and SANG-HAN LEE ${ }^{1,2}$ \\ ${ }^{1}$ Food and Bio-Industry Research Institute and ${ }^{2}$ Department of Food Science and Biotechnology, Kyungpook \\ National University, Daegu 702-701; ${ }^{3}$ Song Kwang Mae Won Co., Ltd., Chilgok 718-852, Republic of Korea
}

Received April 28, 2011; Accepted June 24, 2011

DOI: 10.3892/ijmm.2011.763

\begin{abstract}
To ascertain whether an aqueous fraction of Perilla frutescens Britton (PfB/af) has advantageous anti-atopic dermatitis activity, we used a 2,4-dinitrofluorobenzene (DNFB)-induced animal model of atopic dermatitis symptoms to investigate the effects of the extract. We performed an ear swelling assay by comparing thickness of the DNFB-induced ear, and measured the numbers of eosinophils as well as total immune cells. We analyzed the expression levels of matrix metalloproteinase (MMP)-9, interleukin (IL)-31 and of the T-bet transcription factor. The results revealed that $\mathrm{PfB} / \mathrm{af}$ $(100 \mu \mathrm{g} / \mathrm{ml})$ exhibited strong anti-atopic dermatitis activity, interceding drastic reduction (35\%) of the immune response, as measured by the thickness of ear epidermis swelling, and resulting in decreased eosinophil levels (73.7\%) in adjacent skin tissues. Collectively, the present results suggest that PfB/ af has potential for mitigation of atopic dermatitis-like symptoms induced by DNFB in the mouse.
\end{abstract}

\section{Introduction}

It is generally accepted that the daily consumption of plantorigin phytochemicals from vegetables, fruits, teas, or herb extracts may regulate the balance of redundant free-radical attacks (1-3). In the course of screening the active constituents of an immunomodulation from natural resources such as food and/or Oriental herb plants, we found that an aqueous fraction of Perilla frutescens Britton (PfB/af) scored as a good candidate by an in vitro assay (data not shown). Perilla frutescens is an ethnopharmacological plant in Korea and Japan, and its

Correspondence to: Professor Sang-Han Lee, Department of Food Science and Biotechnology, Kyungpook National University, Daegu 702-701, Republic of Korea

E-mail: sang@knu.ac.kr

Present address: ${ }^{4}$ Pharmacology Research Center, Korea Research Institute of Chemical Technology, Daejeon 305-600, Republic of Korea

*Contributed equally

Key words: Perilla frutescens Britton, anti-atopic, matrix metalloproteinase-9, interleukin-31, T-bet leaf has been used as a food source such as MaeSilJangAhJji (in Korea) and Umeboshi (in Japan), which have anti-bacterial activities, and traditional medicine for colds, headaches and body aches, as a constituent of PaeDokSan in Korea. It has also been reported that the aqueous fraction of Perilla frutescens has potent anti-oxidant activity in vitro and in vivo when used as a hepatoprotective agent in an animal model $(4,5)$.

On the other hand, atopic dermatitis is one of the foremost degenerative diseases, which presents as an unceasing inflammatory skin condition with a malfunctioning skin barrier (6). It is now well assumed that some proteases, including matrix metalloproteinases (MMPs), play a pivotal role in the immunohistological and clinical symptoms during the development of atopic dermatitis $(7,8)$. With a few exceptions, classical skin without drastic damage does not exhibit MMP activities; therefore, the comparison of their enzyme activities is a vital index for the alleviation of an allergic reaction (9). However, there are only a few investigations on atopic-related disorders treated with a natural compounds/extracts, and it has been our main goal to investigate the biological effects of anti-atopic dermatitis activity by PfB/af.

In this study, we initially examined the ameliorating effects of PfB/af against 2,4-dinitrofluorobenzene (DNFB)-induced atopic dermatitis in mice. Various biochemical analyses, such as cytokine expressions, and phenotypic atopic symptoms were assessed in order to prove whether $\mathrm{PfB} / \mathrm{af}$ has potential in alleviating the symptoms.

\section{Materials and methods}

Chemicals.Interleukin(IL)-31, T-bet, and MMP-9 antibody were obtained from AnaSpec (no. 54582, Fremont, CA), Santa Cruz Biotechnology (no. 4B10, Santa Cruz, CA), and Cell Signaling (no. 3852, Danvers, MA), respectively. 2,4-Dinitrofluorobenzene (DNFB), formalin, hydrogen peroxide (all from Sigma-Aldrich, St. Louis, MO), and all other chemicals were commercially available.

Animals and care. C57BL/6 mice (6-7 weeks of age, male) were purchased from Samtaco Korea (Osan, Korea). They were housed in an air-conditioned animal room at a temperature of $22 \pm 1^{\circ} \mathrm{C}$ and with a humidity of $65 \pm 5 \%$. All the procedures were performed in compliance with the Guiding Principles for the Care, and Use of Animals and with the in-house guidelines 
of the University (10), and the guidelines of the International Association for the Study of Pain Committee for Research and Ethical Issues (11). We strictly adhered to the rules and in-house guidelines for animal experiments. Animals were allowed to adapt to the laboratory atmosphere for at least 1 week prior to the experiments. The number of animals in each experimental group was limited to seven.

Preparation and fractionation of samples. The leaves of Perilla frutescens Britton were harvested from a farm of Song Kwang Mae Won Co., Chilgok, Korea between September and November, 2008. Leaves $(100 \mathrm{~g})$ were combined with a 3 -fold volume of distilled water $(200 \mathrm{ml})$, and extracted with an extractor (DWP-3800T; DaeWoong Co., Korea). The extracts were filtrated, lyophilized, and finally yielded a $1.3 \%$ aqueous extract $(1.1 \mathrm{~g} / 85 \mathrm{ml})$. The residue was then dissolved in appropriate buffers and adjusted to a concentration of $100 \mathrm{mg} / \mathrm{ml}$, for further investigation (12). The fraction was used in various in vitro and in vivo assays. The voucher specimen of the plant has been deposited in the Lab of Food Enzyme Biotechnology, KNU.

DNFB-induced animal model. Mice were sensitized with DNFB and challenged as previously described (13). Briefly, the mice were soaked with $50 \mu 1$ of $0.5 \%$ DNFB solution onto their clipped abdominal skin for their first sensitization. A cutaneous reaction was evoked in the ear skin by repeated applications with $20 \mu \mathrm{l}$ of $0.2 \%$ DNFB solution. The DNFB challenge was repeated four times every three days for 2 weeks, starting at 5 days after the initial sensitization (Days 5, 8, 11 and 14). The vehicle mice were similarly treated with acetone without DNFB. The fraction was administered 8 times (Days 6, 7, 9, $10,12,13,15$ and 16) once a day for 2 weeks from the day after the first DNFB re-sensitization (Day 6), during the DNFB re-challenge.

Immunohistochemistry. Ear tissues were fixed for $24 \mathrm{~h}$ in a $10 \%$ neutral-buffered formalin solution and routinely processed as described elsewhere (12-14). In brief, paraffin sections were placed on Probe-On slides and incubated with methanol containing a $3 \%$ hydrogen peroxide solution, to inhibit endogenous peroxidase activity. The tissue sections were treated with a $10 \%$ normal goat serum for $1 \mathrm{~h}$ at room temperature, in order to block non-specific binding. The slides were subsequently incubated overnight at $4^{\circ} \mathrm{C}$ with rabbit antimouse MMP-9, IL-31 or T-bet antibodies.

Statistical analysis. Data are represented as the mean \pm standard deviation for the mean values. The statistical significance was determined by the Student's t-test or ANOVA test with independent means, by using a Microsoft Excel program (15). The critical level for significance was set at $\mathrm{P}<0.05$.

\section{Results and Discussion}

Perilla frutescens was originally known as a food-friendly plant, because the leaf was used for anti-bacterial purposes against oral pathogenic bacteria (16). The plant's ripe fruit exhibits potential for ameliorating colds, coughs, insomnia, nerve system weakness and inflammation of various disor-

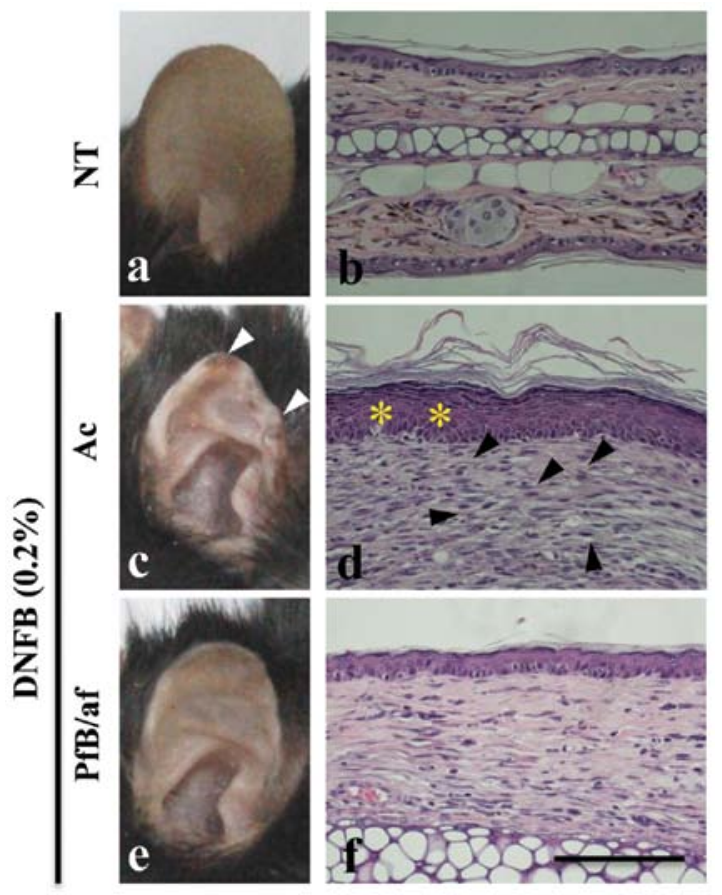

Figure 1. Effect of an aqueous fraction of Perilla frutescens Britton (PfB/af) on DNFB-induced ear swelling in C57BL/6 mice. A DNFB solution, an inducer of a chemically allergic reaction, was applied to a segment of clipped mouse abdominal skin. After initial sensitization, a cutaneous reaction was induced in the skin of the ear by repeated applications of $50 \mu 1$ amounts of $0.2 \%$ (w/v) DNFB solution on four occasions every 3 days for 2 weeks, beginning at 5 days after initial sensitization. Control vehicle mice were similarly treated with acetone (without DNFB). PfB/af was used at a concentration of $100 \mu \mathrm{g} / \mathrm{ml}$. (a, c and e) Clinical features of ear swelling as an index of the immune response, with (e) and without (c) PfB/af treatment after DNFB sensitization. (b, d and f) H\&E staining exhibits the immune events in bulged tissues with (f) or without (d) sample treatment. DNFB-treated ( $\mathrm{d}$ and $\mathrm{f}$ ) and not treated groups (b). Asterisks indicates H\&E-positive cells. Scale bar, $50 \mu \mathrm{m}$.

ders (16-18). It has been reported that the constituents of the aqueous fraction of Perilla frutescens ( $\mathrm{PfB} / \mathrm{af}$ ) exhibit in vitro antioxidant and free radical scavenging behaviors. They also include potent activities for antimicrobial, antitumor, antiviral, and anti-diabetic effects in vitro or in vivo (19-22). However, up to the present time, our knowledge has not gleaned sufficient data concerning its biological activities, and it has never been investigated whether or not $\mathrm{PfB} / \mathrm{af}$ can be useful as an active whole food mixture in a DNFB-induced atopic dermatitis animal model.

In the present study, we evaluated the protective potential of PfB/af on exposure to DNFB, which is well recognized to induce allergic dermatitis in mice skin. First, we assessed whether the PfB/af could be effective on an atopic condition that is induced by DNFB. We, therefore, carried out a histopathological analysis to compare the inner immune events with the DNFB-induced atopic lesions. We cut the ear tissues, sectioned them, and applied histochemical staining to evaluate the numbers of immune cells during DNFB-induced inflammation. Immunohistochemical analyses revealed that $\mathrm{PfB} / \mathrm{af}$ has the potential of alleviating DNFB-induced atopic symptoms in mice. It was shown that DNFB re-sensitization $(0.2 \%)$ brought on severe scarring and eczema on the ear skin (Fig. 1c and d, arrowheads), but the treatment of $\mathrm{PfB} / \mathrm{af}(100 \mu \mathrm{g} / \mathrm{ml})$ led to a 
a

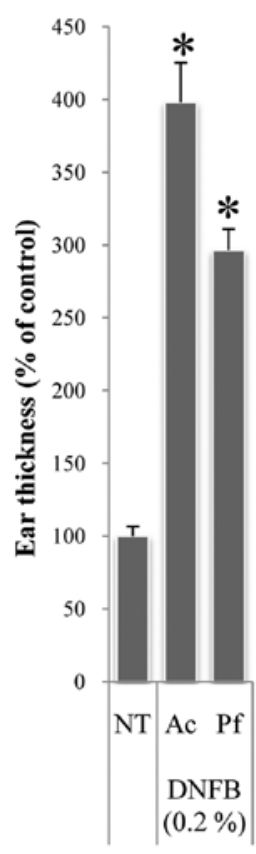

b

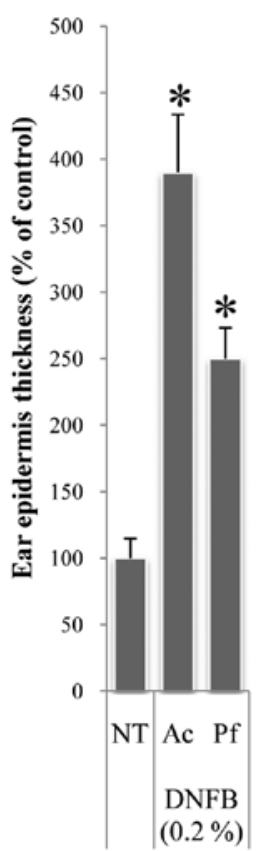

c

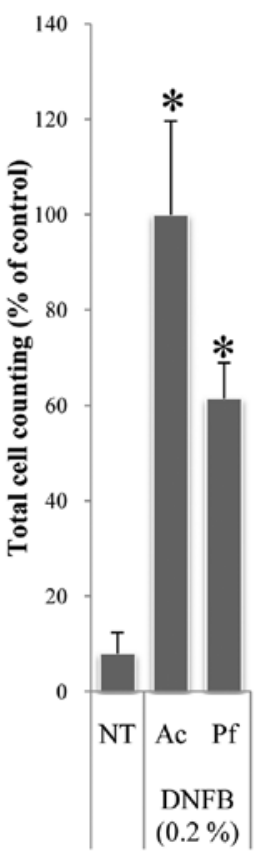

d

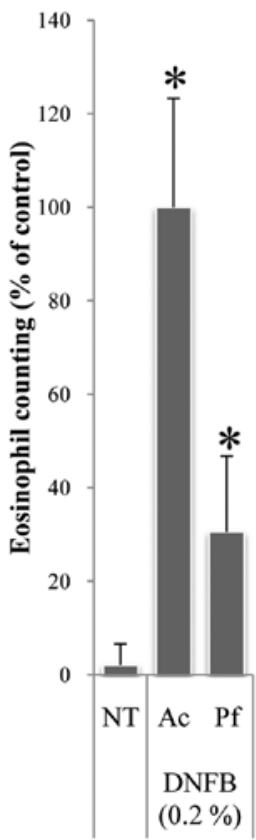

Figure 2. Comparison of atopic dermatitis-related phenotypic factors with or without application of an aqueous fraction of Perilla frutescens Britton (PfB/af). DNFB-treated process was carried out as described in Fig. 1. Cells were photographed under a phase contrast microscope (Nikon, TE-U1000, Tokyo, Japan). Data are expressed as means \pm standard deviations $(\mathrm{n}=5) ;{ }^{*} \mathrm{P}<0.05$. Scale bar, $25 \mu \mathrm{m}$; NT, not treated; Ac, DNFB alone dissolved in acetone; Pf, aqueous fraction of Perilla frutescens Britton.

recovery and alleviated the classical symptoms of atopic dermatitis (Fig. 1e and f), when compared to the control (Fig. 1a and b). To confirm the protective effects of the extract, we measured the ear swelling thickness (Fig. 2a and b). As shown in Figs. 1b and $2 \mathrm{a}$, the ear thickness of control mice was $167.7 \pm 19.7 \mu \mathrm{m}$, and DNFB treatment triggered ear swelling, exhibited by a 3.9-fold increase in swelling (Figs. 1d and 2a). However, the swelling was significantly decreased, to a $35 \%$ level in the ear epidermis thickness within the control group (Figs. If and 2b), by the application of $\mathrm{PfB} / \mathrm{af}$. This result indicates that the $\mathrm{PfB} /$ af treatment reduced the ear swelling thickness by regulating the dermal microenvironment.

The control mice have a classical ear thickness and their clinical observations were identified without any abnormalities (Fig. 1a and b). When the DNFB treatment enlarged the inflammation and broadened their thickness by expressing clear scars and eczema (Fig. 1c and d), the evident inflammatory stained cells increased in number (arrowheads in Fig. 1d). On the contrary, the expressions of inflammation-related cells were markedly reduced by the treatment of PfB/af $(100 \mu \mathrm{g} / \mathrm{ml})$, which is shown in the H\&E staining (magnification, $x 400$; Fig. 1e and f). Total cell counting revealed that the inflammatory cells, by induction of DNFB, marked a 16.6-fold increase in number, whereas that of the PfB/af-treated group was decreased to $58.4 \%$ of the control (Fig. 2c). The numbers of eosinophils were $10.6 \pm 2.3$ cells/block in the DNFB alone group, and 3.0 \pm 1.5 cells/block in the PfB/af-treated group (Fig. 2d). This $73.7 \%$ of decrease in the level of eosinophil numbers may be associated with the active constituents of $\mathrm{PfB} / \mathrm{af}$ with inhibitory activity (Fig. 2d).

The balance between MMPs is largely responsible for the remodelling of skin tissues (9). Various pathological conditions are caused by the unbalanced relationship between MMPs and their inhibitors around the skin's boundary. In both conditions, it appears that the erosion or eczema of skin epithelium is associated with an overexpression of MMP-1, -3, -8, -9 and/ or $-13(9,10)$. In particular, there are certain data that MMP-8 and MMP-9 play mostly a pivotal role in skin remodeling and re-construction during a chemically-induced inflammatory response. Therefore, we examined whether PfB/af affects MMP activity by immunohistochemistry. Fig. 3g-i, illustrates the effects of the DNFB-induced atopic trigger on the expression of MMP-9. Interestingly, the MMP-9 expression levels of the PfB/af treatment $(100 \mu \mathrm{g} / \mathrm{ml})$ were markedly reduced as compared with that of the DNFB-induced group (compare $h$ and i), suggesting that PfB/af can inhibit DNFB-induced skin erosion (Fig. 4c).

It is now recognized that IL-31, preferentially produced by $\mathrm{T}$ helper type 2 cells, is over-expressed with severe pruritus, alopecia, and skin lesions $(23,24)$. Furthermore, the IL-31 receptor expression was increased in diseased tissues derived from an animal model of allergic dermatitis and airway hypersensitivity (25). The above facts led us to examine whether the presence of PfB/af can ameliorate skin lesions. As shown in Fig. 3a-c, we clearly witnessed a decrease of IL-31 positive cells, which was scored approximately at $19.9 \%$ of DNFB alone (Fig. 4a). On the other hand, T-bet exhibited reverse effects. Convincing data can be obtained from T-bet positive cells (Fig. 3d-f). We have already made a stable cell line harboring T-bet promoter genes, in order to select some hits to activate the promoter gene, resulting in a regulated balance between Th1 and Th2 cells (26). Positively in our intention, within the T-bet promoter assay, PfB/af-induced a 1.5 -fold increase in the gene expression at $300 \mu \mathrm{g} / \mathrm{ml}$ (data not shown). Moreover, the T-bet protein expression was also 


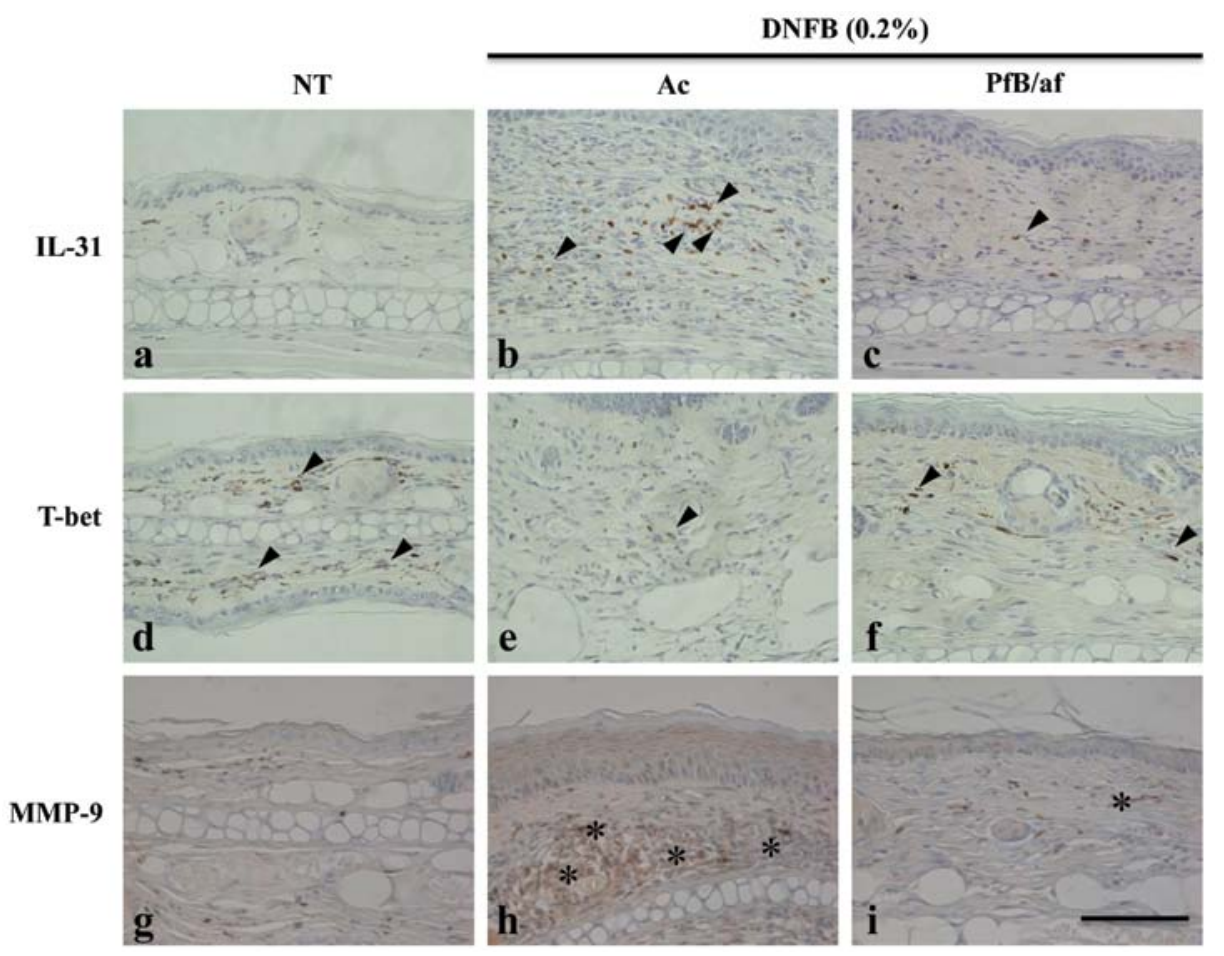

Figure 3. Comparison of atopic dermatitis-related biomarker expression with or without application of an aqueous fraction of Perilla frutescens Britton (PfB/af). The expression levels of IL-31, and T-bet, or MMP-9 positive cells in DNFB-treated mice, either exposed to PfB/af (c, f and i) or not (a, b, d, e, $g$ and $\mathrm{h}$ ), were assayed by immunohistochemical analysis as described in 'Materials and methods'. Arrowheads indicate cells expressing the designated proteins (b, c, d, e and f). Data shown are representative of the features of each group of 7 mice. Scale bar, $25 \mu \mathrm{m}$.
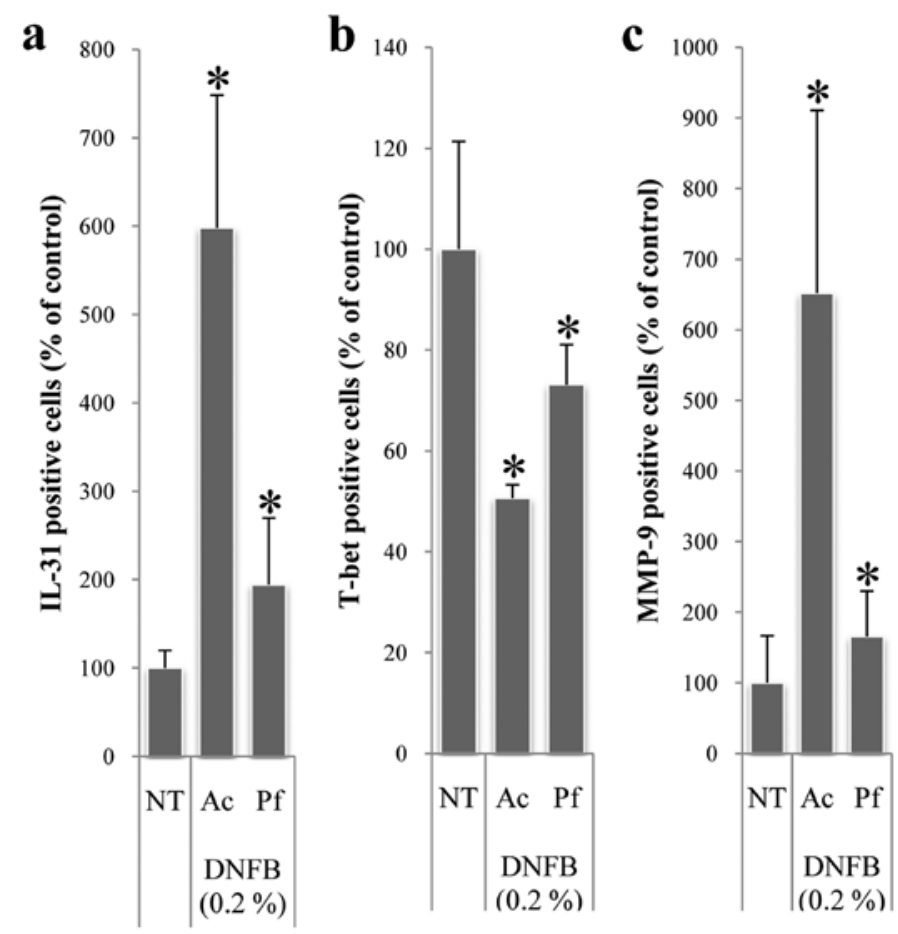

Figure 4. Analysis of atopic dermatitis-related biomarker expressions with or without application of an aqueous fraction of Perilla frutescens Britton (PfB/af). The measurement of IL-31 (a), and T-bet (b), or MMP-9 (c) responsive cells in DNFB-treated mice or not was assayed as described in 'Materials and methods'. Data are the means \pm standard deviations of three independent areas of Fig. 3 data. ${ }^{*}<<0.05$. NT, not treated; Ac, DNFB alone dissolved in acetone; Pf, aqueous fraction of Perilla frutescens Britton.

augmented with a 1.4-fold increase from the PfB/af treatment (Figs. 3d-f and 4b). These results indicate that PfB/af regulated the biomarkers regarding atopic dermatitis by balancing Th1 and Th 2 cells. The T-bet gene is a unique transcription factor which is expressed during Th1 and Th 2 cell differentiation, that was identified from asthmatic symptoms in children $(27,28)$. 
Therefore, it is probable that anti-asthmatic compounds/extracts from Helianthus annuus, Camellia sinensis, Angelica archangelica, or Spinacia oleracea may exhibit potential in mitigating atopic effects in mice $(10,25,28,29)$.

In conclusion, we proved that PfB/af inhibited DNFBinduced atopic inflammation by alleviating MMP-9 and IL-31, but also augmenting T-bet activity. The present data show that $\mathrm{PfB} /$ af has potential in controlling the atopic molecular markers by balancing the Th cell subsets. If we can efficiently modulate the anti-atopic activity by controlling the atopicrelated molecular marker(s), a precise preventive medication as a whole food against atopic dermatitis could be adopted.

\section{Acknowledgements}

This study was supported by a grant of Ministry of Knowledge and Economy, Republic of Korea (S.-H.L. and M.S.S.).

\section{References}

1. Ahmad I, Mehmood Z and Mohammad F: Screening of some Indian medicinal plants for their antimicrobial properties. J Ethnopharmacol 62: 183-193, 1998.

2. Srikumar R, Parthasarathy NJ, Shankar EM, Manikandan S, Vijayakumar R, Thangaraj R, Vijayananth K, Sheeladevi R and Rao UA: Evaluation of the growth inhibitory activities of Triphala against common bacterial isolates from HIV infected patients. Phytother Res 21: 476-480, 2007.

3. Holst B and Williamson G: Nutrients and phytochemicals: from bioavailability to bioefficacy beyond antioxidants. Curr Opin Biotechnol 19: 73-82, 2008.

4. Zekonis G, Zekonis J, Sadzeviciene R, Simoniene G and Kevelaitis E: Effect of Perilla frutescens aqueous extract on free radical production by human neutrophil leukocytes. Medicina 44: 699-705, 2008

5. Kim MK, Lee HS, Kim EJ, Won NH, Chi YM, Kim BC and Lee KW: Protective effect of aqueous extract of Perilla frutescens on tert-butyl hydroperoxide-induced oxidative hepatotoxicity in rats. Food Chem Toxicol 45: 1738-1744, 2007.

6. Boguniewicz M and Leung DY: Recent insights into atopic dermatitis and implications for management of infectious complications. J Allergy Clin Immunol 125: 4-13, 2010.

7. Hanifin J M: Evolving concepts of pathogenesis in atopic dermatitis and other eczemas. J Invest Dermatol 129: 320-322, 2009.

8. Harper JI, Godwin H, Green A, Wilkes LE, Holden NJ, Moffatt M, Cookson WO, Layton G and Chandler S: A study of matrix metalloproteinase expression and activity in atopic dermatitis using a novel skin wash sampling assay for functional biomarker analysis. Br J Dermatol 162: 397-403, 2010.

9. Kähäri VM and Saarialho KU: Matrix metalloproteinases in skin. Exp Dermatol 6: 199-213, 1997.

10. Heo JC, Rho JR, Kim TH, Kim SY and Lee SH: An aqueous extract of green tea Camellia sinensis increases expression of Th1 cell-specific anti-asthmatic markers. Int J Mol Med 22: 763-767, 2008.

11. Charlton E: Ethical guidelines for pain research in humans. Committee on Ethical Issues of the International Association for the Study of Pain. Pain 63: 277-278, 1995.

12. Inagaki N, Shiraishi N, Igeta K, Itoh T, Chikumoto T, Nagao M, Kim JF and Nagai H: Inhibition of scratching behavior associated with allergic dermatitis in mice by tacrolimus, but not by dexamethasone. Eur J Pharmacol 546: 189-196, 2006.
13. Parks WC, Wilson CL and López-Boado YS: Matrix metalloproteinases as modulators of inflammation and innate immunity. Nat Rev Immunol 4: 617-629, 2004.

14. Kim DY, Ryu SY, Lim JE, Lee YS and Ro JY: Anti-inflammatory mechanism of simvastatin in mouse allergic asthma model. Eur J Pharmacol 557: 76-86, 2007.

15. Kwon KH, Kim KI, Jun WJ, Shin DH, Cho HY and Hong BS: In vitro and in vivo effects of macrophage-stimulatory polysaccharide from leaves of Perilla frutescens var. crispa. Biol Pharm Bull 25: 367-371, 2002.

16. Yamamoto $\mathrm{H}$ and Ogawa $\mathrm{T}$ : Antimicrobial activity of perilla seed polyphenols against oral pathogenic bacteria. Biosci Biotechnol Biochem 66: 921-924, 2002.

17. Makino T, Ono T, Muso E and Honda G: Effect of Perilla frutescens on nitric oxide production and DNA synthesis in cultured murine vascular smooth muscle cells. Phytother Res 16: 19-23, 2002.

18. Ueda H, Yamazaki C and Yamazaki M: Luteolin as an antiinflammatory and anti-allergic constituent of Perilla frutescens. Biol Pharm Bull 25: 1197-1202, 2002.

19. Shin TY, Kim SH, Kim YK, Park HJ, Chae BS and Jung HJ: Inhibitory effect of mast cell-mediated immediate-type allergic reactions in rats by Perilla frutescens. Immunopharmacol Immunotoxicol 22: 489-500, 2000.

20. Makino T, Furuta Y, Wakushima H, Fujii H, Saito K and Kano Y: Anti-allergic effect of Perilla frutescens and its active constituents. Phytother Res 17: 240-243, 2003.

21. Banno N, Akihisa T, Tokuda H, Yasukawa K, Higashihara H, Ukiya M, Watanabe K, Kimura Y, Hasegawa $\mathbf{J}$ and Nishino $\mathrm{H}$ : Triterpene acids from the leaves of Perilla frutescens and their anti-inflammatory and antitumor-promoting effects. Biosci Biotechnol Biochem 68: 85-90, 2004.

22. Fujita T, Ohira K, Miyatake K, Nakano $Y$ and Nakayama M: Inhibitory effects of perillosides $\mathrm{A}$ and $\mathrm{C}$, and related monoterpene glucosides on aldose reductase and their structure-activity relationships. Chem Pharm Bull 43: 920-926, 1995.

23. Dillon S R, Sprecher C, Hammond A, Bilsborough J, Rosenfeld FM, Presnell SR, Haugen HS, Maurer M, Harder B, Johnston J, Bort S, Mudri S, Kuijper JL, Bukowski T, Shea P, Dong DL, Dasovich M, Grant FJ, Lockwood L, Levin SD, LeCiel C, Waggie K, Day H, Topouzis S, Kramer J, Kuestner R, Chen Z, Foster D and ParrishNovak J and Gross JA: Interleukin 31, a cytokine produced by activated $\mathrm{T}$ cells, induces dermatitis in mice. Nat Immunol 5: 752-760, 2004.

24. Takaoka A, Arai I, Sugimoto M, Yamaguchi A, Tanaka M and Nakaike S: Expression of IL-31 gene transcripts in NC/Nga mice with atopic dermatitis. Eur J Pharmacol 516: 180-181, 2005.

25. Heo JC, Woo SU, Kweon MA, Park JY, Lee HK, Son M, Rho JR and Lee SH: Aqueous extract of the Helianthus annuus seed alleviates asthmatic symptoms in vivo. Int J Mol Med 21: 57-61, 2008.

26. Szabo SJ, Kim ST, Costa GL, Zhang X, Fathman CG and Glimcher LH: A novel transcription factor, T-bet, directs Th1 lineage commitment. Cell 100: 655-669, 2000.

27. Lametschwandtner G, Biedermann T, Schwärzler C, Günther C, Kund J, Fassl S, Hinteregger S, Carballido PN, Szabo SJ, Glimcher LH and Carballido JM: Sustained T-bet expression confers polarized human TH2 cells with TH1-like cytokine production and migratory capacities. J Allergy Clin Immunol 113: 987-994, 2004.

28. Heo JC and Lee SH: Amelioration of asthmatic-related symptoms by an aqueous extract of Angelica archangelica L. J Life Sci 18: 1336-1341, 2008

29. Heo JC, Park CH, Lee HJ, Kim SI, Kim TH and Lee SH: Amelioration of asthmatic inflammation by an aqueous extract of Spinacia oleracea Linn. Int J Mol Med 25: 409-414, 2010. 